\title{
Vascular Pathology and Blood-Brain Barrier Disruption in Cognitive and Psychiatric Complications of Type 2 Diabetes Mellitus
}

\author{
Yonatan Serlin, ${ }^{1}$ Jaime Levy, $^{2}$ and Hadar Shalev ${ }^{3}$ \\ ${ }^{1}$ Department of Physiology and Neurobiology, Faculty of Health Sciences, Ben-Gurion University of the Negev, \\ Beer-Sheva 84105, Israel \\ ${ }^{2}$ Department of Ophthalmology, Soroka University Medical Center, Ben-Gurion University of the Negev, Beer Sheva 84105, Israel \\ ${ }^{3}$ Department of Psychiatry, Soroka University Medical Center, Ben-Gurion University of the Negev, Beer-Sheva 84105, Israel
}

Correspondence should be addressed to Hadar Shalev, shalev@bgu.ac.il

Received 20 October 2010; Accepted 28 December 2010

Academic Editor: Daniela Kaufer

Copyright () 2011 Yonatan Serlin et al. This is an open access article distributed under the Creative Commons Attribution License, which permits unrestricted use, distribution, and reproduction in any medium, provided the original work is properly cited.

\begin{abstract}
Vascular pathology is recognized as a principle insult in type 2 diabetes mellitus (T2DM). Co-morbidities such as structural brain abnormalities, cognitive, learning and memory deficits are also prevailing in T2DM patients. We previously suggested that microvascular pathologies involving blood-brain barrier (BBB) breakdown results in leakage of serum-derived components into the brain parenchyma, leading to neuronal dysfunction manifested as psychiatric illnesses. The current postulate focuses on the molecular mechanisms controlling BBB permeability in T2DM, as key contributors to the pathogenesis of mental disorders in patients. Revealing the mechanisms underlying BBB dysfunction and inflammatory response in T2DM and their role in metabolic disturbances, abnormal neurovascular coupling and neuronal plasticity, would contribute to the understanding of the mechanisms underlying psychopathologies in diabetic patients. Establishing this link would offer new targets for future therapeutic interventions.
\end{abstract}

\section{Introduction: The Vascular Hypothesis}

Macro- and microvascular complications involving endothelial dysfunction are central to the pathogenesis and clinical manifestations of type 2 diabetes mellitus (T2DM) [1]. Structural brain abnormalities [2-7] and cognitive, learning and memory deficits were demonstrated in T2DM patients [8-10]. We recently published a hypothesis paper suggesting that a primary vascular pathology involving inflammatory cascade and Blood-Brain Barrier (BBB) breakdown, will result in the leakage of serum-derived vascular components into the brain tissue and may cause brain dysfunction which, under some conditions (extent, duration, and/or location), will result in disturbed thinking processes, mood, and behavior, such as those characterizing psychiatric illnesses [11]. The current postulate focuses on inflammation and molecular mechanisms controlling BBB permeability in T2DM as key contributors to the pathogenesis of mental disorders in diabetic patients and suggests novel targets for the prevention and treatment of cognitive and psychiatric complications.

\section{Type 2 Diabetes Mellitus and Vascular Pathology}

T2DM is a multifactorial metabolic disorder. The underlying etiology, pathophysiology and complications of diabetes are still being elucidated (for review see [12]). T2DM is characterized by chronic abnormal high blood glucose levels (hyperglycemia), insulin resistance, and a relative insulin secretion defect [13]. Induction of insulin resistance is linked to obesity and activation of neuroendocrine and inflammatory responses [14-16]. Approximately 200 million people worldwide have diabetes and it is estimated that without proper measures to slow the epidemic advance of the disease, by 2025 the number of patients will increase to 333 million [17]. T2DM is recognized as an independent risk 
factor for cardiovascular disease (CVD), presenting increased risk of morbidity and mortality from coronary heart disease, congestive heart failure, and stroke [18]. Accumulating clinical data disclose the central role of vascular lesions and inflammation in the pathogenesis of T2DM and associated complications [19]. Diabetic macrovascular complications involve vessel obstructions, such as coronary artery diseases, atherosclerosis, and peripheral vascular diseases. Microvascular pathologies include retinopathy, nephropathy, and neuropathy [20]. Direct damage to small blood vessels, particularly by hyperglycemia, is manifested by endothelial dysfunction, diminished perfusion, abnormal endothelial cell (EC) proliferation and increased vessels permeability [21]. T2DM patients exhibit similar microvascular damage within the central nervous system (CNS) which may result in increased incidence of cognitive deterioration, vascular dementia, lacunar infarcts, hemorrhages and Alzheimer's disease [22].

\section{Structure and Function of the Blood-Brain Barrier and Quantification of Its Disruption}

First evidences for a barrier preventing the passage of watersoluble dyes from the circulation to the brain tissue and the spinal cord were presented consecutively by Ehrlich, Goldmann and Lewandowsky in the beginning of the 20th century [23]. At the interfaces between the blood and the neural tissue or its fluid spaces exist three barrier layers: (1) the BBB present in the capillaries throughout the brain, formed by highly specialized EC partitioning between the blood and brain interstitial fluid, (2) the choroid plexus epithelium between blood and ventricular cerebrospinal fluid (CSF), and (3) the arachnoid epithelium between the blood and subarachnoid CSF [24]. The BBB components include the EC with their basement membrane, lining the lumen of brain capillaries. EC adjoined by specific protein tight junctions (e.g., claudins, occludins, $\mathrm{ZO}-1, \mathrm{ZO}-2, \mathrm{ZO}-3$ and cingulin) and display specific transport mechanisms and pinocytic vesicles (for review see [25]). The endothelium is enclosed by brain pericytes and astroglial foot processes which form a third continuous layer that separates these blood vessels from the brain tissue. Jointly, these components form a barrier that hinders the entry of most molecules into the brain, and enable active transportation of penetrated molecules out of the brain. Common brain imaging methods, such as magnetic resonance imaging (MRI), computerized tomography (CT), and single photon emission CT (SPECT) are employed for qualitative evaluation of $\mathrm{BBB}$ disruption in patients. Extravascular accumulation of a peripherally administrated nonpermeable contrast agents, indicate BBB breakdown [11]. Several methods for quantification of BBB permeability using dynamic contrast enhanced imaging were developed, although a routine clinical examination is not yet available [26-28]. In the clinical setting, quantitative evaluation of BBB disruption can be held by CSF analysis for serum proteins (e.g., albumin) or plasma analysis of brain constituents (e.g., S100B-brain-specific astrocytic calciumbinding protein) [29].

\section{Mechanisms of BBB Breakdown}

BBB integrity is altered in diverse pathological conditions. Changes are manifested by disruption of junctional components which result in transbarrier leakage, and $\mathrm{BBB}$ activation, which relates to the expression and secretion of immune factors by its cellular components. The underlying molecular changes leading to BBB dysfunction are not completely clear, but may involve amplification of endothelial caveolae leading to transcytosis of plasma proteins [30, 31], decreased expression of junctional adhesion as well as tight junction proteins [32, 33], and increased expression of matrix metalloproteases [34]. Reactive cellular activity in the neurovascular junction has also been observed, including increase in migratory activity of pericytes [35] and the proliferation of blood vessels due to upregulation of vascular endothelial growth factor (VEGF) [36]. BBB opening itself leads to the exposure of the brain tissue to serum-derived (normally nonpermeable) molecules, which serve as signaling mediators for brain repair mechanisms but may also facilitate BBB breakdown. Agents released during inflammation aggravate the penetrability of the brain endothelium. EC bradykinin B2 receptors activation lead to an increase in intracellular $\mathrm{Ca}^{+}$concentrations [37] and subsequently to activation of endothelial nitric oxide synthase (eNOS) which promotes transient tight junctions opening and increased permeability [38]. Furthermore, bradykinin can activate NF- $\kappa$ B pathway in astrocytes, leading to the release of interleukin-6 (IL-6), which can amplify the effect by acting back on the endothelium [39]. Tumor necrosis factor- $\alpha$ (TNF $\alpha$ ) increases BBB permeability by direct action on the endothelium [40] and indirectly via endothelin- 1 production and IL- $1 \beta$ release from astrocytes [41]. Mediators released from central and peripheral cellular components and connective tissue following injury, can also affect $\mathrm{BBB}$ permeability. For example, histamine and $\mathrm{TNF} \alpha$ and interferon $-\gamma$ released in inflammatory pain can alter brain endothelial permeability [42]. IL- $1 \beta$ release may lead to a decreased concentration or altered localization of the tight junction protein occludin, and thus increases BBB permeability. Metalloproteases causing BBB breakdown are upregulated and released during spreading neuronal depolarization after massive neuronal activation [43].

\section{Blood-Brain Barrier Breakdown in Diabetes Mellitus}

5.1. Anatomical Changes. Altered BBB structure in diabetic patients is a matter of debate. Several studies have indicated that the BBB integrity is sustained in DM, while others revealed association between DM and increased BBB permeability. Intravital microscopy examination of BBB integrity in diabetic rats using fluorescent-labeled albumin displayed intact BBB [44]. These findings should be interpreted with caution since intravital microscopy for quantification of ligands extravasations through the $\mathrm{BBB}$ is often complicated and not specific [45]. Postmortem examination of prefrontal and temporal cortex of diabetic patients together 
with immunohistochemical stainings against serum proteins concluded that the BBB is well maintained [46]. In contrast, growing body of evidence propose an opposing notion. Animal models of ischemic injury in diabetic rats demonstrated that hyperglycemia significantly aggravated BBB permeability, edema formation, and neurological manifestations $[47,48]$. BBB breakdown after ischemia/reperfusion injury result in extravasation of inflammatory cells and fluid into the brain tissue, and thus suggest that BBB disruption has important role in the pathogenesis of brain damage associated with systemic hyperglycemia. MRI brain imaging following intravenous gadolinium administration identified increased BBB permeability in diabetic patients compared to controls [49]. Antibodies against serum S100B and NSE (CNS proteins) were found to be significantly increased in both type 1 and type 2 diabetic subjects compared to controls, implying that diabetes in humans may be associated with alterations in the integrity of the BBB [50].

5.2. Metabolic Changes. Normal metabolic activity of neural tissue relies on constant glucose delivery. Due to the high metabolic demands, glucose transport from the blood across the $\mathrm{BBB}$ into the cells of the brain is mediated by rapid facilitated transport. Glucose transporter proteins (GLUT), particularly GLUT1 and GLUT3 ensure glucose supply. GLUT1 protein is highly expressed at the BBB and GLUT3 is primarily found in neurons. GLUT1 expression is controlled by blood glucose levels, to maintain sufficient distribution for optimal neuronal function [51]. In diabetes, imbalance of glucose metabolism, lead to alterations of glucose transport into the brain. Pardrdige et al. (1990) [52] showed a decrease in GLUT1 expression and activity in diabetic rats thus leading to reduced glucose transport in uncontrolled diabetes. Studies focused on chronic hyperglycemia and increased vascular damage showed that abnormal glucose metabolism results in generation of reactive oxygen species (ROS) followed by oxidative stress, mitochondrial dysfunction and inflammatory response $[53,54]$. Hyperglycemia is presumed to play a role in the generation of acute phase proteins and inflammatory response [55]. It correlates with data about reduction of the levels of acute-phase serum proteins by treatments that increase insulin sensitivity and lower blood glucose [56].

\subsection{Inflammatory Mechanism: From Diabetic Retinopathy to Brain Pathology}

5.3.1. Inflammatory Mechanisms. Inflammatory mechanisms underlying vascular pathology in DM are possibly common to the vasculature in the periphery and CNS. Formation of advanced glycation end products (AGEs) via glycation of blood proteins is a consequence of hyperglycemia, and it results in decreased kidney function and small vessels pathology. AGEs accumulation may induce vascular inflammation by the interactions between AGEs and AGE-specific receptors (RAGE) [57]. AGEs activation of endothelial RAGE promotes upregulation of endothelial adhesion molecules including vascular cell adhesion molecule 1 (VCAM-1) and activates transcription factor nuclear factor- $\kappa \mathrm{B}(\mathrm{NF} \kappa \mathrm{B})$. The former increases monocyte adhesiveness and vascular permeability while the latter regulates multiple proinflammatory and proatherosclerotic target genes in endothelial and vascular smooth muscle cells as well as in macrophages [58].

5.3.2. Diabetic Retinopathy. Well-established data about retinal vessels pathology in DM is available. Due to the structural similarities between the $\mathrm{BBB}$ and the blood-retinal barrier (BRB) and the fact that disruption of the BRB in diabetes is associated with retinopathy, it is logical to assume that altered $\mathrm{BBB}$ function in DM patients may also result in brain pathology. Chronic hyperglycemia, hyperlipidemia, and hypertension contribute to the pathogenesis of Diabetic Retinopathy (DR) [59-61]. Diabetic macular edema (DME) found in $29 \%$ of patients who had diabetes for $\geq 20$ years [62] and is caused by increased level of mediators responsible for retinal vascular permeability as IL-6 and VEGF. These factors promote leakage of intravascular fluid from retinal capillaries into retinal spaces [63]. Further damage arises from retinal EC exposure to AGEs leading to abnormal eNOS expression [64] and induction of VEGF expression [65]. Diabetes may also involve altered retinal blood flow as an outcome of the damage to pericytes enclosing the $\operatorname{BRB}[66,67]$ and correlates with microaneurysm formation. Capillary nonperfusion, EC damage, and vessel occlusions contribute to the retinal microcirculation damage [68]. Capillary occlusion by leukostasis, adherence to the vascular endothelium and cellular degeneration lead to retinal ischemia that stimulates pathologic neovascularization mediated by angiogenic factors (e.g., VEGF) which enhance BRB permeability and result in proliferative diabetic retinopathy (PDR) $[69,70]$. During the last years, anti-VEGF drugs, such as ranibizumab (Lucentis) and bevacizumab (Avastin) are injected into the vitreous for the treatment of diabetic macular edema.

5.3.3. BRB Examination as a Window for BBB Condition. As previously described, the vascular hypothesis speculates that $\mathrm{BBB}$ disintegration may be involved in the pathogenesis of brain diseases. Diabetes-induced microangiopathy of the kidney and retina are well described in the literature. The detailed pathogenesis of microvascular damage within the CNS is less known, since altered functions of cerebral vessels is concealed and less predictable, while in other tissues vascular impairments are detectable and obvious [71]. The analogy between the $\mathrm{BBB}$ and the $\mathrm{BRB}$ is the platform for conceptualization that retinal vessels examination can provide a tool for estimation of cerebral vessels status. In the clinical setting, investigation and documentation of the BRB integrity are held routinely in T2DM patients. Ophthalmic fluorescein angiography (FA) includes intravenously administration of fluorescein producing angiographic display that is used to visualize retinal blood flow dynamics while recording the integrity of the BRB. Correlation between FA results and $\mathrm{BBB}$ permeability measures utilizing dynamic contrast enhanced imaging (e.g., $[26,72]$ ) is thus essential, in order to point out the mutual relation between the two systems. An important feature should be the ability to quantify BRB 
leakage in T2DM patients, and novel imaging methods can be implemented [73]. Future perspectives should focus on developing novel applicable tool for prediction of $\mathrm{BBB}$ breakdown via BRB image analysis. A similar conclusion was published recently, following the results of a prospective study using MRI examination and retinal imaging [74]. It has been shown that retinal microvascular abnormalities are associated with emergence of subclinical brain infarcts and white matter lesions, and proposed that retinal vascular imaging may offer a noninvasive tool to investigate cerebral small-vessel disease.

\section{Blood-Brain Barrier Breakdown in Psychiatric Diseases}

There are evidences linking psychiatric illness with BBB alterations. Quantitative evaluation of BBB disruption utilizes CSF analysis for presence of serum proteins leaked through a permeable barrier, or plasma analysis for molecules found exclusively in the brain (as S100B). Similarly, increase in plasma levels of $\mathrm{S} 100 \mathrm{~B}$ may reflect increased $\mathrm{BBB}$ permeability [29]. CSF/serum albumin ratio was elevated in patients suffering from dementias, in comparison to nondemented individuals [75] and in elderly depressed women compared to women without depression [76]. BBB dysfunction was also shown in schizophrenic patients by measuring increased albumin and IgG CSF levels, with additional correlation between the negative symptomatology to $\mathrm{CSF} /$ serum albumin ratio $[77,78]$. Bell and Zlokovic (2009) recently reviewed the knowledge about the relation between cerebrovascular dysfunction as BBB disruption and neurovascular uncoupling, to cognitive decline and neurodegenerative changes of Alzheimer's disease [79]. Clinical studies demonstrated increased S100B levels in the serum of patients suffering from acute or chronic schizophrenia [80]. Same serum S100B elevation was observed in patients with major depression, with decrease in serum S100B levels during clinical improvement after antidepressant treatment [81].

\section{Comorbidity between Diabetes Mellitus and Psychiatric Disorders}

Among DM patients there is a significant and consistent association with presence of elevated depressive symptoms and the prevalence of major depression, compared with the general population [82-85]. Recently published data shows that higher $\mathrm{A} 1 \mathrm{C}$ levels are associated with lower cognitive function in individuals with diabetes [86]. Accumulating evidence $[9,10]$ indicates that in diabetic patients, hyperglycemia and diabetes durations contribute to brain atrophy and increases the risk of cognitive impairment. Increased expression of RAGE in Alzheimer's disease brain, indicates its relevancy in the pathogenesis of neuronal dysfunction and death [87]. Postmortem studies of individuals with Alzheimer's disease attributes to this opinion by demonstrating AGEs within the senile plaques [88, 89]. Indeed, studies suggest that T2DM is associated with an increased risk of Alzheimer disease, vascular dementia and risk for development of cognitive impairment in comparison with the general population [90-92]. Anxiety disorders were also found in high prevalence in diabetic population $[93,94]$.

\section{Inflammation and Psychopathology}

Inflammatory processes are central to the pathogenesis of T2DM and contribute to BBB dysfunction. Apart from the pathogenic role of the immune responses, accumulating data indicates that immunologic responses also play a role in depression, neurodegeneration, and deficits in cognitive function. Evidence of an inflammatory response in major depression is present over the last two decades [95]. Recent meta-analysis of 24 studies reinforced the notion about cytokine involvement in depression through activation of the inflammatory response [96]. A thorough review by Maes et al. (2009) [97] elaborates the involvement of inflammatory pathways in depression. Increase in proinflammatory cytokines, such as IL- $1 \beta$, IL-6, interferon- $\gamma$ and TNF $\alpha$, with a relative shortage in the anti-inflammatory cytokine IL-10 was documented in depression. Cytokines produced in the periphery and by neurons and glial cells within the CNS are presumed to be involved in the complex autonomic, neuroendocrine, metabolic and behavioral responses to brain injuries as inflammation, ischemia and stroke [98100]. As mentioned previously, in T2DM, inflammation of adipose tissue contributes to insulin resistance. Activated macrophages in the adipose tissue are the primary cellular source of proinflammatory cytokines as IL- $1 \beta$, TNF- $\alpha$ and IL-6. These mediators provide additional links between the participation of immune reaction in T2DM and the brain response. In brain regions lacking intact $\mathrm{BBB}$ (i.e., circumventricular organs), cytokines leakage from the blood into the brain parenchyma may lead to activation of macrophages and induction of a proinflammatory cascade. Additionally, without crossing the $\mathrm{BBB}$, cytokines are able to interact with perivascular macrophages (reviewed by [101]). Clinical data from patients with major depression demonstrate increase of inflammatory features among them [102]. Studies pointing out the existence of positive correlations between plasma concentrations of inflammatory mediators and the severity of depressive symptoms are also available $[103,104]$. Proinflammatory response induces decreased neurogenesis in depression, which is characterized by decreased brain-derived neurotrophic factor (BDNF), neural cell adhesion molecule (NCAM) and fibroblast growth factor (FGF) [105-107]. Inflammation stimulates release or production of corticotropin releasing hormone (CRF), adrenocorticotropic hormone (ACTH) and cortisol via activation of the hypothalamic-pituitary-adrenal axis (HPA) and cortisol in turn may participate in neural atrophy $[108,109]$. Furthermore, inflammatory cytokines as IL$1 \beta, \operatorname{IFN} \gamma$, and TNF $\alpha$ cause induction of indoleamine-2,3dioxygenase (IDO), an enzyme catabolizing tryptophan into neurotoxic metabolites known as TRYCATs. IDO activation is significantly related to inflammatory signs and to the severity of depressive symptoms $[110,111]$. Serotonin levels 
are affected by inflammation since tryptophan is the precursor of 5-HT. IDO metabolize tryptophan in the kynurenine pathway and therefore less tryptophan is available to synthesize 5-HT. Activation of the brain's microglia by Th1 cytokines, either secreted from activated astrocytes or from the periphery, induces IDO and may thus reduce 5HT levels and result in depression. Astrocytic activation in the brain, facilitated by BBB disruption in inflammatory condition of T2DM may also alter network properties and neuronal excitability by changing glutamate levels and affecting synaptic plasticity. Cytokines may generate, through the kynurenine pathway, the formation of quinolinic acid-an NMDA receptor (NMDAR) agonist. Microglia are the only cells in the CNS that express the complete enzymatic pathway required for the synthesis of quinolinic acid [112]. Hence, inflammatory mediators acting on microglia will increase the levels of quinolinic acid and will activate NMDA receptors. These findings match with new evidence suggesting that heightened glutamate receptor activity in major depression, can underlie inflammation-associated depressive disorders [113]. In addition, quinolinic acid directly causes release of glutamate [114]. Thus, inflammatory mediators can lead to an environment of excess glutamate. Glutamate receptor activation enhances the effect of $\mathrm{BBB}$ breakdown by induction of astrocytic transformation. A vicious cycle of cytokine secretion, microglial activation, and further enhancement of glutamate receptors activation is created (see below). Activated microglial cells are also key contributors to the inflammatory response which occur during chronic neurodegeneration in diseases such as Alzheimer's disease, prion disease and Parkinson's disease [115]. These activated microglia release proinflammatory cytokines which affect injured neurons and may exacerbate lesion size and neuronal loss. Postmortem examination of brain tissue from patients suffered from Alzheimer's disease revealed large numbers of activated microglia associated with the amyloid deposits and in regions of the brain where there is neuronal loss [116]. Metabolic syndrome, T2DM, and decline in cognitive function share common inflammatory markers [117]. Elevated levels of insulin may lead to cognitive decline via the effect of hyperinsulinemia on neuronal metabolism and reduced clearance of $\beta$ amyloid, a frequent pathologic feature of obesity, metabolic syndrome, DM, and Alzheimer's disease [118]. A Recent study showed decrease in executive and processing function among metabolic syndrome patients [119]. Moreover, patients with impaired insulin function were found to have lower levels of the neurotrophic protein BDNF. Decreases in hippocampal BDNF levels showed association with stress-induced depressive behaviors and conversely, antidepressant treatment enhanced the expression of BDNF [120].

\section{BBB Breakdown and Psychopathology}

Neuropsychiatric disorders such as depression, mood and anxiety disorders, are associated with cerebrovascular impairments [121]. BBB breakdown will result in induction of signaling pathways leading to transformation and activa- tion of the surroundingcells. We mentioned previously how local inflammatory brain responses following BBB changes influence endothelial and glial cells towards elevation of cytokine expression. It is possible to assume that glial cell activation will also participate in the functional changes occurring in the vascular environment and the adjacent neuropil. Indeed, compromised $\mathrm{BBB}$ results in a rapid transformation of the resting astrocytes into their active form in ischemic, inflammatory and traumatic brain injuries. Astrocytic endfeet are considered an integral part of the $\mathrm{BBB}$ and surround capillaries in the CNS to regulate the vascular tone [122] and tight junction expression [123]. Experimental evidence suggests that upon BBB breakdown, infiltration of albumin, the most abundant serum protein, into the neuropil may account for the astrocytic transformation via the transforming growth factor beta (TGF $\beta$ ) signaling pathway. Transformed astrocytes undergoes modification in gene expression that includes the upregulation of GFAP and S100B, downregulation of glutamate transporters, glutamine synthase and the inward rectifying potassium channel $\left(\mathrm{K}_{\mathrm{IR} 4.1}\right), \mathrm{AQP} 4$ and gap junctions' proteins [124, 125]. The subsequent gene expression affects the extracellular environment through increased concentrations of potassium and glutamate causing amplification of neuronal excitability [126]. The participation of calcium metabolism in neurovascular coupling provides a hint for a possible pathologic molecular mechanism that may arise from astrocytic activation. Neuron-to-astrocyte signaling is considered being a key mechanism in functional hyperemia. The resultant increase in extracellular glutamate following astrocytic transformation can activate glutamate receptors (mGluRs) located on astrocytes. It has been shown that the dilation of arterioles triggered by neuronal activity is dependent on glutamate-mediated cytosolic calcium $\left(\left[\mathrm{Ca}^{2+}\right] \mathrm{i}\right)$ oscillations in astrocytes [127]. Activation of mGluRs and the subsequent elevation in $\left[\mathrm{Ca}^{2+}\right] \mathrm{i}$ in astrocytes ultimately creates $\left[\mathrm{Ca}^{2+}\right] \mathrm{i}$ increase in the endfeet [128]. Zonta et al. [127] demonstrated that inhibition of astrocytic $\mathrm{Ca}^{2+}$ responses resulted in the impairment of activity-dependent vasodilation, whereas selective activation of single astrocytes in close proximity to arterioles triggered vessel relaxation [127]. They further observed that in vivo blockade of glutamatemediated $\left[\mathrm{Ca}^{2+}\right]$ i elevations in astrocytes reduced hyperemic reaction in the somatosensory cortex during contralateral forepaw stimulation. Excess of extracellular glutamate that leads to activation of mGluRs and the increase of $\left[\mathrm{Ca}^{2+}\right] \mathrm{i}$ in the endfeet, initiate the activation of $\mathrm{Ca}^{2+}$-sensitive $\mathrm{K}^{+}$ channels (BK) and the efflux of $\mathrm{K}^{+}$. BK channels were proposed to play a role in the $\mathrm{K}^{+}$modulation of cerebral blood flow [129]. Extracellular excess of potassium has the potential to generate changes in the vascular tone through activation of inward rectifying $\mathrm{K}^{+}$channels $\left(\mathrm{K}_{\mathrm{IR}}\right)$ located in smooth muscle (SMC) layer of vessel [130]. BK channels, expressed abundantly in astrocytic endfeets, exhibit sensitivity to membrane depolarization and intracellular calcium levels. Neuronal stimulation of brain slices produced BK channel-mediated $\mathrm{K}^{+}$release in astrocytic endfeets, altered the extracellular $\mathrm{K}^{+}\left(\left[\mathrm{K}^{+}\right] \mathrm{o}\right)$ level in the perivascular space and generated a signal that produces vasodilatory response 
by $\mathrm{K}_{\mathrm{IR}}$ channels in parenchymal arteriole SMC. The elevation of $\left[\mathrm{K}^{+}\right]$o from $3 \mathrm{mmol} / \mathrm{L}$ to $8 \mathrm{mmol} / \mathrm{L}$ hyperpolarizes parenchymal arteriolar membranes from -45 to $-80 \mathrm{mV}$, and causes a rapid and profound dilation of isolated pressurized parenchymal arterioles as well as arterioles in brain slices [131]. Thus, astrocytic activation after BBB disruption, subsequent reduction in $\mathrm{K}^{+}$buffering and the increase of extracellular glutamate and $\mathrm{K}^{+}$, elevates the $\left[\mathrm{K}^{+}\right]$o levels. This will consequent in enhancement of the mutual activity of glutamate-mediated $\left[\mathrm{Ca}^{2+}\right] \mathrm{i}$ oscillations in astrocytes, BK activation and vasodilatation through $\mathrm{SMC} \mathrm{K}^{+}$channels. A direct link between the metabolic state in the brain tissue and astrocyte signaling was recently established [132]. According to our hypothesis, the changes in the perivascular microenvironment and the metabolic dysregulation arising from impairment in cerebrovascular response as disturbed or extensive hyperemia may take part in the mechanisms underlying brain pathologies. Hyperemia in the active regions and hypoperfusion of surrounding areas, under some conditions (extent, duration and location) may result in impaired metabolism, inadequate homeostasis preservation, formation of reactive oxygen species and insufficient removal of toxic metabolites. These insults may participate in the evolvement of cognitive or psychiatric illnesses. Mechanisms of abnormal plasticity are also suspected to participate in the development of mental disturbances following BBB breakdown, glial activation and inflammation, via the effect of excess of glutamate. We hypothesize that diffusion of glutamate and $\mathrm{K}^{+}$out of the narrow synaptic cleft will affect neighboring synapses, resulting in a loss of synapseand pathway-specific plasticity. Astrocyte-mediated plasticity mechanisms utilize glutamate for transient mGluRdependent neuromodulation. In addition long-term potentiation via NMDAR-independent mechanism showing $\mathrm{Ca}_{2}{ }^{+}$ elevation in astrocytes that modulates transmitter release probability and evokes long-term synaptic plasticity [133]. This control in transmitter release at the synapse and the strengthening of synaptic connectivity may possibly result in synaptic tuning in circuits involved in cognitive processing and the control of limbic system excitability [134]. Formation of new synapses may reduce specificity and is expected to activate larger neuronal networks in response to stimuli. These alterations might be expressed in disturbed thinking processes and extreme mood-related behavioral responses, depending on the involved network.

\section{Conclusion}

Inflammation and vascular pathology have a significant contribution for the pathogenesis of T2DM complications. Neuropsychiatric disorders are also associated with inflammatory reaction and cerebrovascular impairments. Brain injuries that often involve BBB breakdown and astrocytic response increase the risk for neuropsychiatric sequelae, including personality changes, depression, anxiety, dementia, and perhaps psychosis $[135,136]$. T2DM patients show higher susceptibility to cerebrovascular diseases which according to our hypothesis may explain the increased incidence of cognitive deterioration, depression, vascular dementia, lacunar infarcts, hemorrhages and Alzheimer's disease among these patients. Revealing the mechanisms underlying the effects of diabetes on BBB structure and function and understanding the role of inflammation, impaired neurovascular coupling, metabolic defects and altered neuronal plasticity in the neuropsychiatric sequela of T2DM, will create a target for clinical and pharmacologic modalities and a potential platform for future therapeutic intervention.

\section{References}

[1] M. Stumvoll, B. J. Goldstein, and T. W. Van Haeften, “Type 2 diabetes: principles of pathogenesis and therapy," Lancet, vol. 365, no. 9467, pp. 1333-1346, 2005.

[2] T. Pirttila, R. Jarvenpaa, P. Laippala, and H. Frey, "Brain atrophy on computerized axial tomography scans: interaction of age, diabetes and general morbidity," Gerontology, vol. 38, no. 5, pp. 285-291, 1992.

[3] Y. Araki, M. Nomura, H. Tanaka et al., "MRI of the brain in diabetes mellitus," Neuroradiology, vol. 36, no. 2, pp. 101103, 1994.

[4] T. Den Heijer, S. E. Vermeer, E. J. Van Dijk et al., “Type 2 diabetes and atrophy of medial temporal lobe structures on brain MRI," Diabetologia, vol. 46, no. 12, pp. 1604-1610, 2003.

[5] B. Van Harten, F. E. De Leeuw, H. C. Weinstein, P. Scheltens, and G. J. Biessels, "Brain imaging in patients with diabetes: a systematic review," Diabetes Care, vol. 29, no. 11, pp. 25392548, 2006.

[6] S. M. Gold, I. Dziobek, V. Sweat et al., "Hippocampal damage and memory impairments as possible early brain complications of type 2 diabetes," Diabetologia, vol. 50, no. 4, pp. 711-719, 2007.

[7] P. L. Yau, D. C. Javier, C. M. Ryan et al., "Preliminary evidence for brain complications in obese adolescents with type 2 diabetes mellitus," Diabetologia, vol. 53, no. 11, pp. 22982306, 2010.

[8] A. M. Abbatecola and G. Paolisso, "Relationship between baseline glycemic control and cognitive function in individuals with type 2 diabetes and other cardiovascular risk factors: the Action to Control Cardiovascular Risk in DiabetesMemory in Diabetes (ACCORD-MIND) Trial," Diabetes Care, vol. 32, no. 8, p. e102, 2009.

[9] D. G. Bruce, W. A. Davis, G. P. Casey et al., "Predictors of cognitive impairment and dementia in older people with diabetes," Diabetologia, vol. 51, no. 2, pp. 241-248, 2008.

[10] A. M. Tiehuis, Y. van der Graaf, F. L. Visseren et al., "Diabetes increases atrophy and vascular lesions on brain MRI in patients with symptomatic arterial disease," Stroke, vol. 39, no. 5, pp. 1600-1603, 2008.

[11] H. Shalev, Y. Serlin, and A. Friedman, "Breaching the bloodbrain barrier as a gate to psychiatric disorder," Cardiovascular Psychiatry and Neurology, vol. 2009, Article ID 278531, 7 pages, 2009.

[12] P. N. Surampudi, J. John-Kalarickal, and V. A. Fonseca, "Emerging concepts in the pathophysiology of type 2 diabetes mellitus," Mount Sinai Journal of Medicine, vol. 76, no. 3, pp. 216-226, 2009.

[13] Y. Lin and Z. Sun, "Current views on type 2 diabetes," Journal of Endocrinology, vol. 204, no. 1, pp. 1-11, 2010.

[14] K. E. Wellen and G. S. Hotamisligil, "Obesity-induced inflammatory changes in adipose tissue," Journal of Clinical Investigation, vol. 112, no. 12, pp. 1785-1788, 2003. 
[15] H. Xu, G. T. Barnes, Q. Yang et al., "Chronic inflammation in fat plays a crucial role in the development of obesity-related insulin resistance," Journal of Clinical Investigation, vol. 112, no. 12, pp. 1821-1830, 2003.

[16] S. H. Golden, M. Lazo, M. Carnethon et al., "Examining a bidirectional association between depressive symptoms and diabetes," Journal of the American Medical Association, vol. 299, no. 23, pp. 2751-2759, 2008.

[17] S. Wild, G. Roglic, A. Green, R. Sicree, and H. King, "Global prevalence of diabetes: estimates for the year 2000 and projections for 2030," Diabetes Care, vol. 27, no. 5, pp. 1047 1053, 2004.

[18] J. B. McGill, "Improving microvascular outcomes in patients with diabetes through management of hypertension," Postgraduate Medicine, vol. 121, no. 2, pp. 89-101, 2009.

[19] P. M. Ridker, "Inflammatory biomarkers and risks of myocardial infarction, stroke, diabetes, and total mortality: implications for longevity," Nutrition Reviews, vol. 65, no. 12, pp. S253-S259, 2007.

[20] P. Geraldes and G. L. King, "Activation of protein kinase C isoforms and its impact on diabetic complications," Circulation Research, vol. 106, no. 8, pp. 1319-1331, 2010.

[21] S. I. Yamagishi and T. Imaizumi, "Diabetic vascular complications: pathophysiology, biochemical basis and potential therapeutic strategy," Current Pharmaceutical Design, vol. 11, no. 18, pp. 2279-2299, 2005.

[22] M. Ristow, "Neurodegenetive disorders associated with diabetes mellitus," Journal of Molecular Medicine, vol. 82, no. 8, pp. 510-529, 2004.

[23] B. T. Hawkins and T. P. Davis, "The blood-brain barrier/neurovascular unit in health and disease," Pharmacological Reviews, vol. 57, no. 2, pp. 173-185, 2005.

[24] N. J. Abbott, L. Ronnback, and E. Hansson, "Astrocyteendothelial interactions at the blood-brain barrier," Nature Reviews Neuroscience, vol. 7, no. 1, pp. 41-53, 2006.

[25] B. V. Zlokovic, "The blood-brain barrier in health and chronic neurodegenerative disorders," Neuron, vol. 57, no. 2, pp. 178-201, 2008.

[26] P. S. Tofts, G. Brix, D. L. Buckley et al., "Estimating kinetic parameters from dynamic contrast-enhanced T-weighted MRI of a diffusable tracer: standardized quantities and symbols," Journal of Magnetic Resonance Imaging, vol. 10, no. 3, pp. 223-232, 1999.

[27] G. Zaharchuk, "Theoretical basis of hemodynamic MR imaging techniques to measure cerebral blood volume, cerebral blood flow, and permeability," American Journal of Neuroradiology, vol. 28, no. 10, pp. 1850-1858, 2007.

[28] O. Tomkins, I. Shelef, I. Kaizerman et al., "Blood-brain barrier disruption in post-traumatic epilepsy," Journal of Neurology, Neurosurgery and Psychiatry, vol. 79, no. 7, pp. 774-777, 2008.

[29] N. Marchi, P. Rasmussen, M. Kapural et al., "Peripheral markers of brain damage and blood-brain barrier dysfunction," Restorative Neurology and Neuroscience, vol. 21, no. 3-4, pp. 109-121, 2003.

[30] S. Nag, R. Venugopalan, and D. J. Stewart, "Increased caveolin-1 expression precedes decreased expression of occludin and claudin-5 during blood-brain barrier breakdown," Acta Neuropathologica, vol. 114, no. 5, pp. 459-469, 2007.

[31] S. Nag, J. L. Manias, and D. J. Stewart, "Expression of endothelial phosphorylated caveolin-1 is increased in brain injury," Neuropathology and Applied Neurobiology, vol. 35, no. 4, pp. 417-426, 2009.
[32] J. Zhao, A. N. Moore, J. B. Redell, and P. K. Dash, "Enhancing expression of Nrf2-driven genes protects the blood-brain barrier after brain injury," Journal of Neuroscience, vol. 27, no. 38, pp. 10240-10248, 2007.

[33] D. Yeung, J. L. Manias, D. J. Stewart, and S. Nag, "Decreased junctional adhesion molecule-A expression during bloodbrain barrier breakdown," Acta Neuropathologica, vol. 115, no. 6, pp. 635-642, 2008.

[34] T. Higashida, C. W. Kreipke, J. A. Rafols et al., "The role of hypoxia-inducible factor-1alpha, aquaporin-4, and matrix metalloproteinase- 9 in blood-brain barrier disruption and brain edema after traumatic brain injury," Journal of Neurosurgery, vol. 114, no. 1, pp. 92-101, 2011.

[35] P. Dore-Duffy, C. Owen, R. Balabanov, S. Murphy, T. Beaumont, and J. A. Rafols, "Pericyte migration from the vascular wall in response to traumatic brain injury," Microvascular Research, vol. 60, no. 1, pp. 55-69, 2000.

[36] S. Nag, J. L. Takahashi, and D. W. Kilty, "Role of vascular endothelial growth factor in blood-brain barrier breakdown and angiogenesis in brain trauma," Journal of Neuropathology and Experimental Neurology, vol. 56, no. 8, pp. 912-921, 1997.

[37] F. Marceau and D. Regoli, "Bradykinin receptor ligands: therapeutic perspectives," Nature Reviews Drug Discovery, vol. 3, no. 10, pp. 845-852, 2004.

[38] L. M. F. Leeb-Lundberg, "Bradykinin specificity and signaling at GPR100 and B kinin receptors," British Journal of Pharmacology, vol. 143, no. 8, pp. 931-932, 2004.

[39] M. Schwaninger, S. Sallmann, N. Petersen et al., "Bradykinin induces interleukin-6 expression in astrocytes through activation of nuclear factor- $\kappa$ B," Journal of Neurochemistry, vol. 73, no. 4, pp. 1461-1466, 1999.

[40] M. A. Deli, L. Descamps, M. P. Dehouck et al., "Exposure of tumor necrosis factor- $\alpha$ to luminal membrane of bovine brain capillary endothelial cells cocultured with astrocytes induces a delayed increase of permeability and cytoplasmic stress fiber formation of actin," Journal of Neuroscience Research, vol. 41, no. 6, pp. 717-726, 1995.

[41] N. Didier, I. A. Romero, C. Créminon, A. Wijkhuisen, J. Grassi, and A. Mabondzo, "Secretion of interleukin- $1 \beta$ by astrocytes mediates endothelin-1 and tumour necrosis factor- $\alpha$ effects on human brain microvascular endothelial cell permeability," Journal of Neurochemistry, vol. 86, no. 1, pp. 246-254, 2003.

[42] J. D. Huber, R. D. Egleton, and T. P. Davis, "Molecular physiology and pathophysiology of tight junctions in the blood -brain barrier," Trends in Neurosciences, vol. 24, no. 12, pp. 719-725, 2001.

[43] Y. Gursoy-Ozdemir, J. Qiu, N. Matsuoka et al., "Cortical spreading depression activates and upregulates MMP-9," Journal of Clinical Investigation, vol. 113, no. 10, pp. 14471455, 2004.

[44] W. G. Mayhan, "Effect of diabetes mellitus on disruption of the blood-brain barrier during acute hypertension," Brain Research, vol. 534, no. 1-2, pp. 106-110, 1990.

[45] M. H. Horani and A. D. Mooradian, "Effect of diabetes on the blood brain barrier," Current Pharmaceutical Design, vol. 9, no. 10, pp. 833-840, 2003.

[46] J. Dai, G. F. J. M. Vrensen, and R. O. Schlingemann, "Bloodbrain barrier integrity is unaltered in human brain cortex with diabetes mellitus," Brain Research, vol. 954, no. 2, pp. 311-316, 2002 
[47] W. D. Dietrich, O. Alonso, and R. Busto, "Moderate hyperglycemia worsens acute blood-brain barrier injury after forebrain ischemia in rats," Stroke, vol. 24, no. 1, pp. 111-116, 1993.

[48] H. Kamada, F. Yu, C. Nito, and P. H. Chan, "Influence of hyperglycemia on oxidative stress and matrix metalloproteinase- 9 activation after focal cerebral ischemia/reperfusion in rats: relation to blood-brain barrier dysfunction," Stroke, vol. 38, no. 3, pp. 1044-1049, 2007.

[49] J. M. Starr, J. M. Wardlaw, K. Ferguson, A. MacLullich, I. J. Deary, and I. Marshall, "Increased blood-brain barrier permeability in type II diabetes demonstrated by gadolinium magnetic resonance imaging," Journal of Neurology Neurosurgery and Psychiatry, vol. 74, no. 1, pp. 70-76, 2003.

[50] M. R. Hovsepyan, M. J. Haas, A. S. Boyajyan et al., "Astrocytic and neuronal biochemical markers in the sera of subjects with diabetes mellitus," Neuroscience Letters, vol. 369, no. 3, pp. 224-227, 2004.

[51] S. J. Vannucci, E. M. Gibbs, and I. A. Simpson, "Glucose utilization and glucose transporter proteins GLUT-1 and GLUT-3 in brains of diabetic $(\mathrm{db} / \mathrm{db})$ mice," American Journal of Physiology, vol. 272, no. 2, pp. E267-E274, 1997.

[52] W. M. Pardridge, D. Triguero, and C. R. Farrell, "Downregulation of blood-brain barrier glucose transporter in experimental diabetes," Diabetes, vol. 39, no. 9, pp. 1040-1044, 1990.

[53] S. Pennathur and J. W. Heinecke, "Oxidative stress and endothelial dysfunction in vascular disease," Current Diabetes Reports, vol. 7, no. 4, pp. 257-264, 2007.

[54] V. B. Schrauwen-Hinderling, M. Roden, M. E. Kooi, M. K. C. Hesselink, and P. Schrauwen, "Muscular mitochondrial dysfunction and type 2 diabetes mellitus," Current Opinion in Clinical Nutrition and Metabolic Care, vol. 10, no. 6, pp. 698-703, 2007.

[55] Y. Lin, M. W. Rajala, J. P. Berger, D. E. Moller, N. Barzilai, and P. E. Scherer, "Hyperglycemia-induced production of acute phase reactants in adipose tissue," Journal of Biological Chemistry, vol. 276, no. 45, pp. 42077-42083, 2001.

[56] C. L. Scott, "Diagnosis, prevention, and intervention for the metabolic syndrome," American Journal of Cardiology, vol. 92, no. 1, pp. 35i-42i, 2003.

[57] R. Meerwaldt, C. J. Zeebregts, G. Navis, J. L. Hillebrands, J. D. Lefrandt, and A. J. Smit, "Accumulation of advanced glycation end products and chronic complications in ESRD treated by dialysis," American Journal of Kidney Diseases, vol. 53, no. 1, pp. 138-150, 2009.

[58] R. Piga, Y. Naito, S. Kokura, O. Handa, and T. Yoshikawa, "Short-term high glucose exposure induces monocyteendothelial cells adhesion and transmigration by increasing VCAM- 1 and MCP-1 expression in human aortic endothelial cells," Atherosclerosis, vol. 193, no. 2, pp. 328-334, 2007.

[59] R. Klein, B. E. K. Klein, S. E. Moss, M. D. Davis, and D. L. DeMets, "Glycosylated hemoglobin predicts the incidence and progression of diabetic retinopathy," Journal of the American Medical Association, vol. 260, no. 19, pp. 2864-2871, 1988.

[60] S. Vitale, M. G. Maguire, R. P. Murphy et al., "Clinically significant macular edema in type I diabetes: incidence and risk factors," Ophthalmology, vol. 102, no. 8, pp. 1170-1176, 1995.

[61] R. Klein, B. E. K. Klein, S. E. Moss, and K. J. Cruickshanks, "The wisconsin epidemiologic study of diabetic retinopathy: XVII. The 14- year incidence and progression of diabetic retinopathy and associated risk factors in type 1 diabetes," Ophthalmology, vol. 105, no. 10, pp. 1801-1815, 1998.
[62] R. Klein, B. E. K. Klein, and S. E. Moss, "The Wisconsin epidemiologic study of diabetic retinopathy. IV. Diabetic macular edema," Ophthalmology, vol. 91, no. 12, pp. 1464-1474, 1984.

[63] C. H. Meyer, "Current treatment approaches in diabetic macular edema," Ophthalmologica, vol. 221, no. 2, pp. 118-131, 2007.

[64] U. Chakravarthy, R. G. Hayes, A. W. Stitt, E. McAuley, and D. B. Archer, "Constitutive nitric oxide synthase expression in retinal vascular endothelial cells is suppressed by high glucose and advanced glycation end products," Diabetes, vol. 47, no. 6, pp. 945-952, 1998.

[65] M. Lu, M. Kuroki, S. Amano et al., "Advanced glycation end products increase retinal vascular endothelial growth factor expression," Journal of Clinical Investigation, vol. 101, no. 6, pp. 1219-1224, 1998.

[66] T. A. Ciulla, A. Harris, P. Latkany et al., "Ocular perfusion abnormalities in diabetes," Acta Ophthalmologica Scandinavica, vol. 80, no. 5, pp. 468-477, 2002.

[67] C. Paget, M. Lecomte, D. Ruggiero, N. Wiernsperger, and M. Lagarde, "Modification of enzymatic antioxidants in retinal microvascular cells by glucose or advanced glycation end products," Free Radical Biology and Medicine, vol. 25, no. 1, pp. 121-129, 1998.

[68] K. Miyamoto and Y. Ogura, "Pathogenetic potential of leukocytes in diabetic retinopathy," Seminars in Ophthalmology, vol. 14, no. 4, pp. 233-239, 1999.

[69] L. P. Aiello, R. L. Avery, P. G. Arrigg et al., "Vascular endothelial growth factor in ocular fluid of patients with diabetic retinopathy and other retinal disorders," New England Journal of Medicine, vol. 331, no. 22, pp. 1480-1487, 1994.

[70] J. W. Miller, A. P. Adamis, and L. P. Aiello, "Vascular endothelial growth factor in ocular neovascularization and proliferative diabetic retinopathy," Diabetes/Metabolism Reviews, vol. 13, no. 1, pp. 37-50, 1997.

[71] J. D. Huber, "Diabetes, cognitive function, and the bloodbrain barrier," Current Pharmaceutical Design, vol. 14, no. 16, pp. 1594-1600, 2008.

[72] O. Tomkins, I. Shelef, I. Kaizerman et al., "Blood-brain barrier disruption in post-traumatic epilepsy," Journal of Neurology, Neurosurgery and Psychiatry, vol. 79, no. 7, pp. 774-777, 2008.

[73] O. Prager, Y. Chassidim, C. Klein, H. Levi, I. Shelef, and A. Friedman, "Dynamic in vivo imaging of cerebral blood flow and blood-brain barrier permeability," NeuroImage, vol. 49, no. 1, pp. 337-344, 2010.

[74] N. Cheung, T. Mosley, A. Islam et al., "Retinal microvascular abnormalities and subclinical magnetic resonance imaging brain infarct: a prospective study," Brain, vol. 133, no. 7, pp. 1987-1993, 2010.

[75] I. Skoog, A. Wallin, P. Fredman et al., "A population study on blood-brain barrier function in 85-year-olds: relation to Alzheimer's disease and vascular dementia," Neurology, vol. 50, no. 4, pp. 966-971, 1998.

[76] P. Gudmundsson, I. Skoog, M. Waern et al., "The relationship between cerebrospinal fluid biomarkers and depression in elderly women," American Journal of Geriatric Psychiatry, vol. 15, no. 10, pp. 832-838, 2007.

[77] N. Muller and M. Ackenheil, "Immunoglobulin and albumin content of cerebrospinal fluid in schizophrenic patients: relationship to negative symptomatology," Schizophrenia Research, vol. 14, no. 3, pp. 223-228, 1995. 
[78] M. J. Schwarz, M. Ackenheil, M. Riedel, and N. Müller, "Blood-cerebrospinal fluid barrier impairment as indicator for an immune process in schizophrenia," Neuroscience Letters, vol. 253, no. 3, pp. 201-203, 1998.

[79] R. D. Bell and B. V. Zlokovic, "Neurovascular mechanisms and blood-brain barrier disorder in Alzheimer's disease," Acta Neuropathologica, vol. 118, no. 1, pp. 103-113, 2009.

[80] M. Rothermundt, G. Ponath, T. Glaser, G. Hetzel, and V. Arolt, "S100B serum levels and long-term improvement of negative symptoms in patients with schizophrenia," Neuropsychopharmacology, vol. 29, no. 5, pp. 1004-1011, 2004.

[81] M. L. Schroeter, H. Abdul-Khaliq, M. Krebs, A. Diefenbacher, and I. E. Blasig, "Serum markers support disease-specific glial pathology in major depression," Journal of Affective Disorders, vol. 111, no. 2-3, pp. 271-280, 2008.

[82] M. De Groot, R. Anderson, K. E. Freedland, R. E. Clouse, and P. J. Lustman, "Association of depression and diabetes complications: a meta-analysis," Psychosomatic Medicine, vol. 63, no. 4, pp. 619-630, 2001.

[83] R. J. Anderson, K. E. Freedland, R. E. Clouse, and P. J. Lustman, "The prevalence of comorbid depression in adults with diabetes: a meta-analysis," Diabetes Care, vol. 24, no. 6, pp. 1069-1078, 2001.

[84] S. H. Saydah, F. L. Brancati, S. H. Golden, J. Fradkin, and M. I. Harris, "Depressive symptoms and the risk of type 2 diabetes mellitus in a US sample," Diabetes/Metabolism Research and Reviews, vol. 19, no. 3, pp. 202-208, 2003.

[85] S. Ali, M. A. Stone, J. L. Peters, M. J. Davies, and K. Khunti, "The prevalence of co-morbid depression in adults with Type 2 diabetes: a systematic review and meta-analysis," Diabetic Medicine, vol. 23, no. 11, pp. 1165-1173, 2006.

[86] T. Cukierman-Yaffe, H. C. Gerstein, J. D. Williamson et al., "Relationship between baseline glycemic control and cognitive function in individuals with type 2 diabetes and other cardiovascular rIsk factors the action to control cardiovascular risk in diabetes-memory in diabetes (ACCORDMIND) trial," Diabetes Care, vol. 32, no. 2, pp. 221-226, 2009.

[87] S. D. Yan, X. Chen, J. Fu et al., "RAGE and amyloid- $\beta$ peptide neurotoxicity in Alzheimer's disease," Nature, vol. 382, no. 6593, pp. 685-691, 1996.

[88] K. Horie, T. Miyata, T. Yasuda et al., "Immunohistochemical localization of advanced glycation end products, pentosidine, and carboxymethyllysine in lipofuscin pigments of Alzheimer's disease and aged neurons," Biochemical and Biophysical Research Communications, vol. 236, no. 2, pp. 327-332, 1997.

[89] H. Vlassara, R. Bucala, and L. Striker, "Pathogenic effects of advanced glycosylation: biochemical, biologic, and clinical implications for diabetes and aging," Laboratory Investigation, vol. 70, no. 2, pp. 138-151, 1994.

[90] E. W. Gregg, K. Yaffe, J. A. Cauley et al., "Is diabetes associated with cognitive impairment and cognitive decline among older women?" Archives of Internal Medicine, vol. 160, no. 2, pp. 174-180, 2000.

[91] K. Yaffe, T. Blackwell, A. M. Kanaya, N. Davidowitz, E. Barrett-Connor, and K. Krueger, "Diabetes, impaired fasting glucose, and development of cognitive impairment in older women," Neurology, vol. 63, no. 4, pp. 658-663, 2004.

[92] K. V. Allen, B. M. Frier, and M. W. J. Strachan, "The relationship between type 2 diabetes and cognitive dysfunction: longitudinal studies and their methodological limitations," European Journal of Pharmacology, vol. 490, no. $1-3$, pp. 169-175, 2004.
[93] C. E. Lloyd, P. H. Dyert, and A. H. Barnett, "Prevalence of symptoms of depression and anxiety in a diabetes clinic population," Diabetic Medicine, vol. 17, no. 3, pp. 198-202, 2000.

[94] R. J. Anderson, M. De Groot, A. B. Grigsby et al., "Anxiety and poor glycemic control: a meta-analytic review of the literature," International Journal of Psychiatry in Medicine, vol. 32, no. 3, pp. 235-247, 2002.

[95] A. Gardner and R. G. Boles, "Beyond the serotonin hypothesis: mitochondria, inflammation and neurodegenera-tion in major depression and affective spectrum disorders," Progress in Neuro-Psychopharmacology and Biological Psychiatry. In press.

[96] Y. Dowlati, N. Herrmann, W. Swardfager et al., "A metaanalysis of cytokines in major depression," Biological Psychiatry, vol. 67, no. 5, pp. 446-457, 2010.

[97] M. Maes, R. Yirmyia, J. Noraberg et al., "The inflammatory \& neurodegenerative (I\&ND) hypothesis of depression: leads for future research and new drug developments in depression," Metabolic Brain Disease, vol. 24, no. 1, pp. 27-53, 2009.

[98] E. M. Sternberg, "Neural-immune interactions in health and disease," Journal of Clinical Investigation, vol. 100, no. 11, pp. 2641-2647, 1997.

[99] C. Woiciechowsky, K. Asadullah, D. Nestler et al., "Sympathetic activation triggers systemic interleukin-10 release in immunodepression induced by brain injury," Nature Medicine, vol. 4, no. 7, pp. 808-813, 1998.

[100] N. Vila, J. Castillo, A. Dávalos, and A. Chamorro, "Proinflammatory cytokines and early neurological worsening in ischemic stroke," Stroke, vol. 31, no. 10, pp. 2325-2329, 2000.

[101] J. C. O'Connor, D. R. Johnson, and G. G. Freund, "Psychoneuroimmune implications of type 2 diabetes: redux," Immunology and Allergy Clinics of North America, vol. 29, no. 2, pp. 339-358, 2009.

[102] C. L. Raison, L. Capuron, and A. H. Miller, "Cytokines sing the blues: inflammation and the pathogenesis of depression," Trends in Immunology, vol. 27, no. 1, pp. 24-31, 2006.

[103] A. J. Thomas, S. Davis, C. Morris, E. Jackson, R. Harrison, and J. T. O'Brien, "Increase in interleukin- $1 \beta$ in late-life depression," American Journal of Psychiatry, vol. 162, no. 1, pp. 175-177, 2005.

[104] S. Alesci, P. E. Martinez, S. Kelkar et al., "Major depression is associated with significant diurnal elevations in plasma interleukin-6 levels, a shift of its circadian rhythm, and loss of physiological complexity in its secretion: clinical implications," Journal of Clinical Endocrinology and Metabolism, vol. 90, no. 5, pp. 2522-2530, 2005.

[105] C. A. Turner, H. Akil, S. J. Watson, and S. J. Evans, "The fibroblast growth factor system and mood disorders," Biological Psychiatry, vol. 59, no. 12, pp. 1128-1135, 2006.

[106] H. D. Schmidt and R. S. Duman, "The role of neurotrophic factors in adult hippocampal neurogenesis, antidepressant treatments and animal models of depressive-like behavior," Behavioural Pharmacology, vol. 18, no. 5-6, pp. 391-418, 2007.

[107] C. Sandi and R. Bisaz, "A model for the involvement of neural cell adhesion molecules in stress-related mood disorders," Neuroendocrinology, vol. 85, no. 3, pp. 158-176, 2007.

[108] M. Maes, E. Bosmans, H. Y. Meltzer, S. Scharpe, and E. Suy, "Interleukin-1 $\beta$ : a putative mediator of HPA axis hyperactivity in major depression?" American Journal of Psychiatry, vol. 150, no. 8, pp. 1189-1193, 1993. 
[109] I. Goshen, T. Kreisel, O. Ben-Menachem-Zidon et al., "Brain interleukin-1 mediates chronic stress-induced depression in mice via adrenocortical activation and hippocampal neurogenesis suppression," Molecular Psychiatry, vol. 13, no. 7, pp. 717-728, 2008.

[110] M. Maes, R. Verkerk, S. Bonaccorso, W. Ombelet, E. Bosmans, and S. Scharpé, "Depressive and anxiety symptoms in the early puerperium are related to increased degradation of tryptophan into kynurenine, a phenomenon which is related to immune activation," Life Sciences, vol. 71, no. 16, pp. 1837-1848, 2002.

[111] M. C. Wichers, G. Kenis, G. H. Koek, G. Robaeys, N. A. Nicolson, and M. Maes, "Interferon- $\alpha$-induced depressive symptoms are related to changes in the cytokine network but not to cortisol," Journal of Psychosomatic Research, vol. 62, no. 2, pp. 207-214, 2007.

[112] R. Dantzer, J. C. O'Connor, G. G. Freund, R. W. Johnson, and K. W. Kelley, "From inflammation to sickness and depression: when the immune system subjugates the brain," Nature Reviews Neuroscience, vol. 9, no. 1, pp. 46-56, 2008.

[113] N. Müller and M. J. Schwarz, "The immune-mediated alteration of serotonin and glutamate: towards an integrated view of depression," Molecular Psychiatry, vol. 12, no. 11, pp. 988-1000, 2007.

[114] E. Fedele and A. C. Foster, "An evaluation of the role of extracellular amino acids in the delayed neurodegeneration induced by quinolinic acid in the rat striatum," Neuroscience, vol. 52, no. 4, pp. 911-917, 1993.

[115] V. H. Perry, "The influence of systemic inflammation on inflammation in the brain: implications for chronic neurodegenerative disease," Brain, Behavior, and Immunity, vol. 18, no. 5, pp. 407-413, 2004.

[116] S. M. Allan and N. J. Rothwell, "Cytokines and acute neurodegeneration," Nature Reviews Neuroscience, vol. 2, no. 10, pp. 734-744, 2001.

[117] S. Craft, "The role of metabolic disorders in Alzheimer disease and vascular dementia: two roads converged," Archives of Neurology, vol. 66, no. 3, pp. 300-305, 2009.

[118] J.-S. Roriz, T. M. Sá-Roriz, I. Rosset et al., “(Pre)diabetes, brain aging, and cognition," Biochimica et Biophysica Acta, vol. 1792, no. 5, pp. 432-443, 2009.

[119] B. Segura, M. A. Jurado, N. Freixenet, C. Albuin, J. Muniesa, and C. Junqué, "Mental slowness and executive dysfunctions in patients with metabolic syndrome," Neuroscience Letters, vol. 462, no. 1, pp. 49-53, 2009.

[120] K. Martinowich, H. Manji, and B. Lu, "New insights into BDNF function in depression and anxiety," Nature Neuroscience, vol. 10, no. 9, pp. 1089-1093, 2007.

[121] E. Chemerinski and S. R. Levine, "Neuropsychiatric disorders following vascular brain injury," Mount Sinai Journal of Medicine, vol. 73, no. 7, pp. 1006-1014, 2006.

[122] K. M. Dunn and M. T. Nelson, "Potassium channels and neurovascular coupling," Circulation Journal, vol. 74, no. 4, pp. 608-616, 2010.

[123] R. F. Haseloff, I. E. Blasig, H. C. Bauer, and H. Bauer, "In search of the astrocytic factor(s) modulating blood-brain barrier functions in brain capillary endothelial cells in vitro," Cellular and Molecular Neurobiology, vol. 25, no. 1, pp. 25-39, 2005.

[124] A. Friedman, D. Kaufer, and U. Heinemann, "Blood-brain barrier breakdown-inducing astrocytic transformation: novel targets for the prevention of epilepsy," Epilepsy Research, vol. 85, no. 2-3, pp. 142-149, 2009.
[125] L. P. Cacheaux, S. Ivens, Y. David et al., "Transcriptome profiling reveals TGF- $\beta$ signaling involvement in epileptogenesis," Journal of Neuroscience, vol. 29, no. 28, pp. 8927-8935, 2009.

[126] S. Ivens, D. Kaufer, L. P. Flores et al., "TGF- $\beta$ receptormediated albumin uptake into astrocytes is involved in neocortical epileptogenesis," Brain, vol. 130, no. 2, pp. 535-547, 2007.

[127] M. Zonta, M. C. Angulo, S. Gobbo et al., "Neuron-toastrocyte signaling is central to the dynamic control of brain microcirculation," Nature Neuroscience, vol. 6, no. 1, pp. 43-50, 2003.

[128] H. R. Parri and V. Crunelli, "The role of $\mathrm{Ca}^{2+}$ in the generation of spontaneous astrocytic $\mathrm{Ca}^{2+}$ oscillations," Neuroscience, vol. 120, no. 4, pp. 979-992, 2003.

[129] D. L. Price, J. W. Ludwig, H. Mi, T. L. Schwarz, and M. H. Ellisman, "Distribution of rSlo $\mathrm{Ca}^{2+}$-activated $\mathrm{K}^{+}$channels in rat astrocyte perivascular endfeet," Brain Research, vol. 956, no. 2, pp. 183-193, 2002.

[130] H. J. Knot, P. A. Zimmermann, and M. T. Nelson, "Extracellular $\mathrm{K}^{+}$-induced hyperpolarizations and dilatations of rat coronary and cerebral arteries involve inward rectifier $\mathrm{K}^{+}$ channels," Journal of Physiology, vol. 492, no. 2, pp. 419-430, 1996.

[131] J. A. Filosa, A. D. Bonev, S. V. Straub et al., "Local potassium signaling couples neuronal activity to vasodilation in the brain," Nature Neuroscience, vol. 9, no. 11, pp. 1397-1403, 2006.

[132] G. Carmignoto and M. Gómez-Gonzalo, "The contribution of astrocyte signalling to neurovascular coupling," Brain Research Reviews, vol. 63, no. 1-2, pp. 138-148, 2010.

[133] G. Perea and A. Araque, "Astrocytes potentiate transmitter release at single hippocampal synapses," Science, vol. 317, no. 5841, pp. 1083-1086, 2007.

[134] P. Jourdain, L. H. Bergersen, K. Bhaukaurally et al., "Glutamate exocytosis from astrocytes controls synaptic strength," Nature Neuroscience, vol. 10, no. 3, pp. 331-339, 2007.

[135] S. Fleminger, "Long-term psychiatric disorders after traumatic brain injury," European Journal of Anaesthesiology, vol. 25, supplement 42, pp. 123-130, 2008.

[136] D. F. Guerreiro, R. Navarro, M. Silva, M. Carvalho, and C. Gois, "Psychosis secondary to traumatic brain injury," Brain Injury, vol. 23, no. 4, pp. 358-361, 2009. 


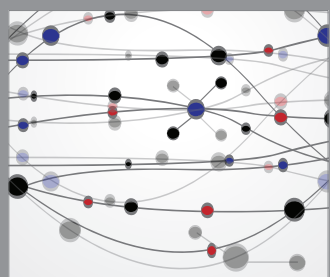

The Scientific World Journal
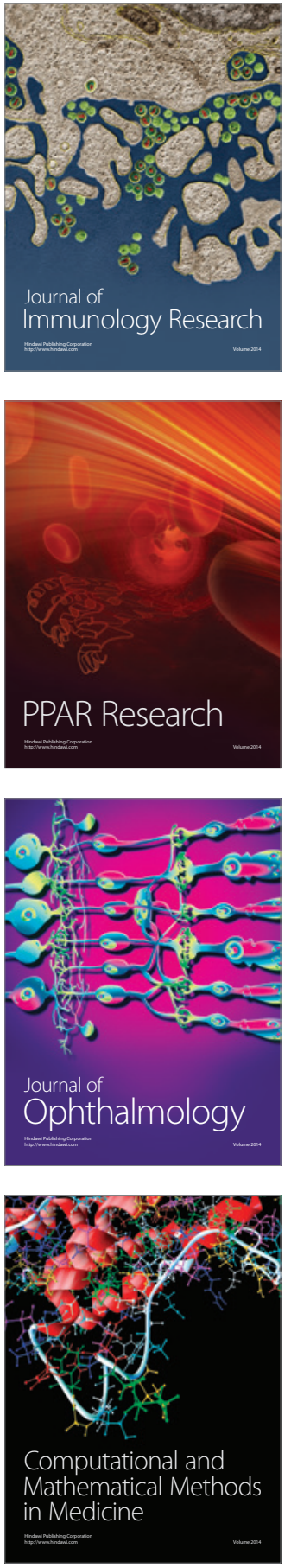

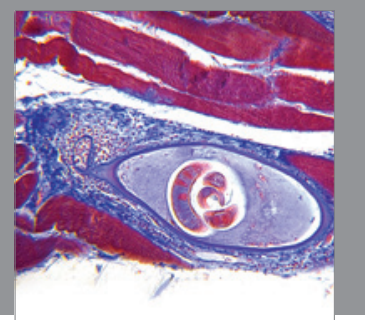

Gastroenterology

Research and Practice
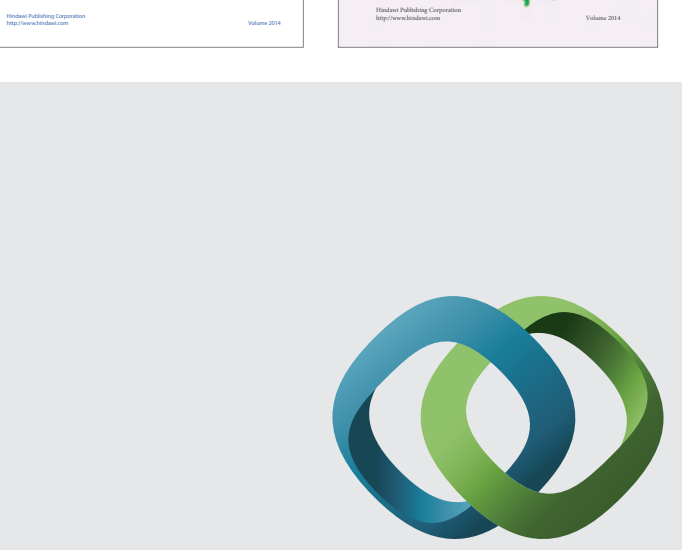

\section{Hindawi}

Submit your manuscripts at

http://www.hindawi.com
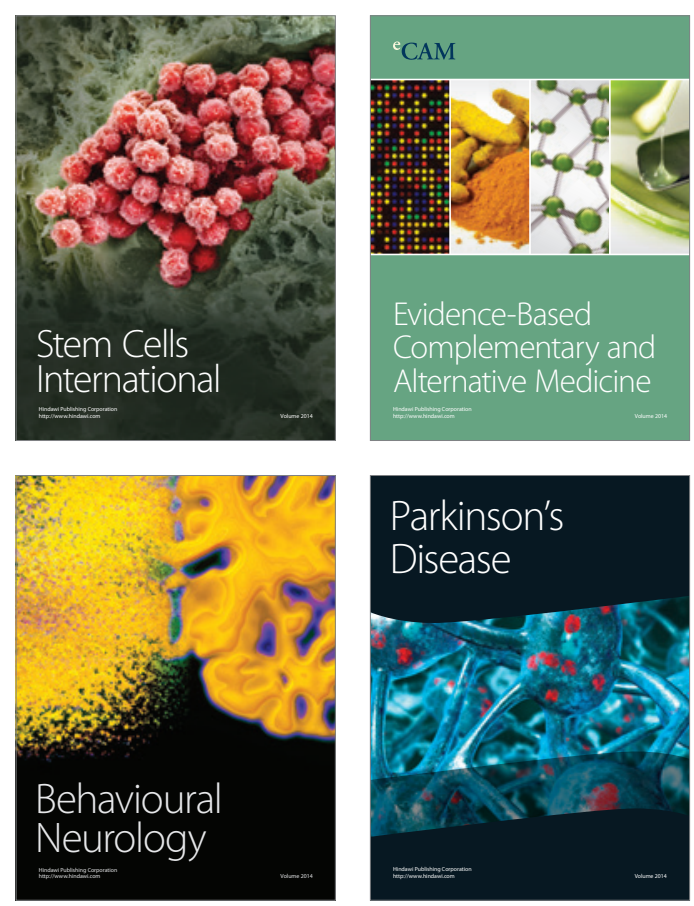

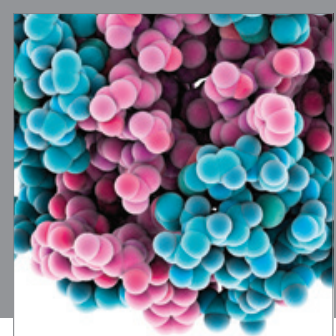

Journal of
Diabetes Research

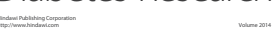

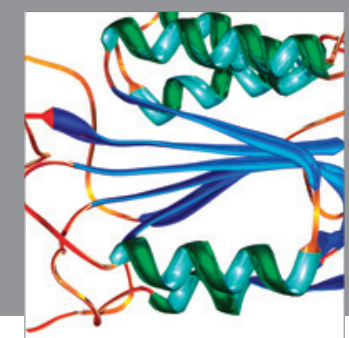

Disease Markers
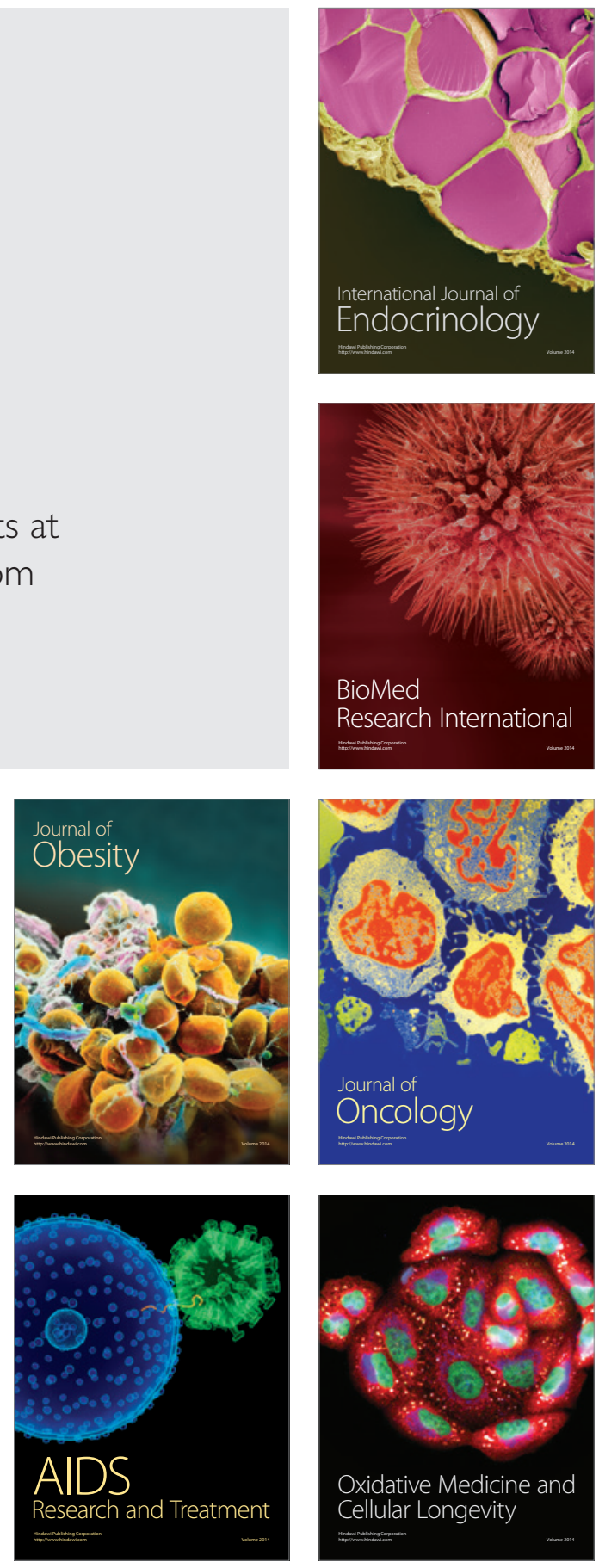